\begin{tabular}{ll}
\hline \hline MINING AND METALLURGY INSTITUTE BOR & ISSN: 2334-8836 (Štampano izdanje) \\
UDK: 622 & ISSN: 2406-1395 (Online) \\
\hline \hline
\end{tabular}

\title{
FRAGMENTING OF CYLINDRICAL ROCK SPECIMENS UNDER EXPLOSIVE LOAD - COMPARISON BETWEEN MODEL AND LABORATORY RESULTS
}

\begin{abstract}
Achieving optimal fragmentation of the blasted rock is common task for mining engineers. During the years many models for fragmentation prediction were developed and are mostly based on empirical relations. While there are theoretical expansions of the empirical models, one could note the lack of the purely theoretical models based on the rock breakage theory. Herein, validation of such model is presented. Model considers only the fragmenting of the cylindrical monolith rock specimens and compares the results with the laboratory tests that were carried out in same manner. Model provides results through the definition of the fragment shape and size and then geometrical fragmenting of the whole specimen. Comparison of the model and laboratory results shows high level of agreement between sieving curves and also confirm the right constitution of the rock blasting theory that this model is based on.

Keywords: rock fragmentation, rock breakage by explosives, fragmentation model, laboratory tests, blasting
\end{abstract}

\section{INTRODUCTION}

Achieving optimal fragmentation of blasted rock is one of the main tasks in mining production. This means that after blasting muck pile will contain minimum possible amount of non-blasted blocks and fines. If these criteria are not satisfied, there is need for secondary fragmenting of large blocks which is time consuming. On the other hand, if there is large amount of fines it could be impossible to load these fractions and it leads to greater loss of blasted material. Kuz-Ram empirical model was the first to provide estimate of the fragment size distribution. This model was proposed by Kuznetsov [1] and is based on the RosinRammler distribution. Later on, model was expanded by Cunningham [2, 3] and up to these days it is the one most used and modi- fied model for fragment size estimation. Many researchers were considering differrent statistical distributions and their application inside the Kuz-Ram model. As for the example, Ouchterlony [4] proposed the Swebrec function that fits the size distribution curve in the domain of fines. Sanchidrián et al. [5, 6] provides summary of many functions used for the description of the fragment size distribution. Djordjevic [7] in his work also considered the fines range that is obviously weak point in existing methodologies. It is very clear that Kuz-Ram model is the central point when it comes to the fragment size estimation. Also, it is noticeable that there is lack of the fundamental models that are based on the rock breakage theories. Although there are considerable researches in the area of the crack propaga-

\footnotetext{
*University of Belgrade, Faculty of Mining and Geology, veljko.lapcevic@rgf.bg.ac.rs
} 


\section{ROCK BLASTING THEORY}

tion and development under the explosive load [8-10], there is no exact method to predict fragment size distribution. One part of the model that is presented and validated herein is based on the actual rock breakage theory and tends to provide exact fragment size and spatial distribution. Model considers idealistic situation where monolith rock specimens are blasted and analyzed for fragment size distribution. Validation of the model is done through the comparison between model and laboratory results.
According to a rock blasting theory [11] it is possible to calculate the radii of zones having a different density of radial cracks around the blasthole. This theory explains the fracturing mechanism of rock under explosive load. Here, the main part of that theory is presented, since it is the basis of this paper. Detonation of an explosive charge in rock results in dynamic loading of the walls of the blasthole and generation of a pressure wave that transmits energy through the surrounding medium.

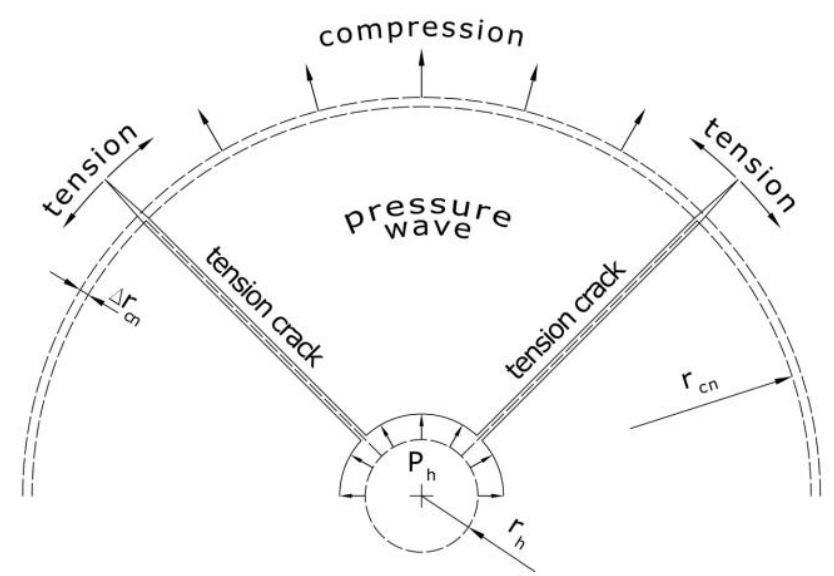

Figure 1 Radial crack formation mechanism

The pressure wave extends from borehole walls circularly around the borehole (Figure 1). At the distance $r_{c n}$ from the borehole compressive stress of the rock in the radial direction is:

$$
\sigma_{r c}=P_{h} \frac{r_{h}}{r_{c n}}
$$

Where:

$\sigma_{r c}$ - radial compressive stress

$P_{h}$ - borehole pressure

$r_{h}$ - borehole radius

$r_{c n}$ - crack zone radius
On the other hand:

$$
\sigma_{r c}=M \cdot e_{r}
$$

Where:

$$
\begin{aligned}
& M=E \cdot \frac{(1-v)}{(1+v)(1-2 v)} \\
& k=\frac{(1-v)}{(1+v)(1-2 v)} \\
& \sigma_{r c}=E \cdot k \cdot e_{r} \\
& M \text { - pressure wave modulus [12] } \\
& e_{r} \text { - radial strain }
\end{aligned}
$$


$E$-Young`s modulus of rock

$v$ - Poisson's ratio

Or:

$e_{r}=\frac{\sigma_{r c}}{E \cdot k}$

Therefore:

$e_{r}=\frac{P_{h} \cdot r_{h}}{E \cdot k \cdot r_{c n}}$

At the distance $r_{c n}$, before the pressure wave gets to it, the perimeter of the closed circular ring zone of rock mass is:

$$
O_{r}=2 \pi r_{c n}
$$

When the pressure wave reaches the closed circular ring zone of rock mass, it is moved to a new position with a radius $\left(r_{c n}+\Delta r_{c n}\right)$, and with the perimeter:

$$
O_{\left(r_{c n}+\Delta r_{c n}\right)}=2 \pi\left(r_{c n}+\Delta r_{c n}\right)
$$

Therefore:

$$
O_{\left(r_{c n}+\Delta r_{c n}\right)}=2 \pi\left(r_{c n}+e_{r} \cdot r_{c n}\right)
$$

Once the closed circular ring zone of rock mass is subjected to tension with a lateral strain:

$$
e_{l}=\frac{O_{\left(r_{c n}+\Delta r_{c n}\right)}-O_{r_{c n}}}{O_{r_{c n}}}=e_{r}
$$

For the formation of the radial tension cracks, tensile strain is required:

$$
e_{t}=\frac{\sigma_{t}}{E}
$$

Where:

$\mathrm{e}_{\mathrm{t}}$ - tensile strain

$\sigma_{t}$ - tensile strength

E - Young`s modulus

In addition, the number $(n)$ of radial tensile cracks formed at a distance $r_{c n}$ will be:

$$
n=\frac{e_{l}}{e_{t}}
$$

Therefore, it is:

$$
n=\frac{P_{h} r_{h}}{k \sigma_{t} r_{c n}}
$$

Therefore:

$$
r_{c n}=\frac{P_{h} r_{h}}{k \sigma_{t} n}
$$

Therefore, for the borehole radius $r_{h}=0.051 \mathrm{~m}$ and the borehole pressure $P_{h}=1.6 \mathrm{GPa}$ in limestone with tensile strength $\sigma_{t}=7 \mathrm{MPa}$, Poisson's ratio $v=0.3$ (Table 1) will be:

Table 1 Zone radius with crack density

\begin{tabular}{cccccc}
\hline \hline $\mathrm{n}$ & 2 & 4 & 8 & 16 & 32 \\
\hline \hline $\mathrm{r}_{\mathrm{cn}}(\mathrm{m})$ & 4.32 & 2.16 & 1.08 & 0.54 & 0.27 \\
\hline
\end{tabular}

In Figure 2, the schematic illustration of tension crack length and density around blast hole is shown. Practical application of this theory was demonstrated for the estimation of blasted rock fragmentation[13] and for the blast damage zone extent estimation [14]. 


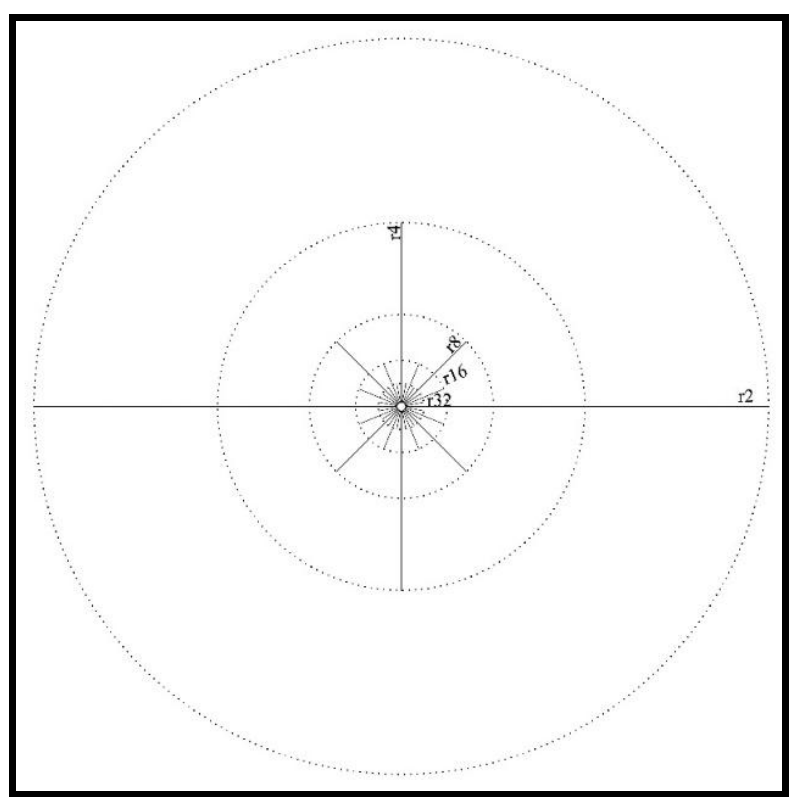

Figure 2 Schematic illustration of tension crack length and density around blasthole

\section{FRAGMENTING OF THE CYLINDRICAL MONOLITH ROCK SPECIMENS UNDER THE EXPLOSIVE LOAD}

Rock blasting theory [11] explains radial tension crack formation under explosive load. This theory was used for the constitution of the model for estimating blasted rock fragmentation [13]. Model describes application of the rock blasting theory for the fragment size estimation after the blasting in the rock mass. It should be mentioned that there is no theoretical explanation of complete rock fragmenting mechanism which may be used for fragment size estimation. Due to the lack of theoretical explanations of rock fragmenting, main assumption is that rock fragments have quasi-isometric dimensions while size distribution is fractal like. In this manner it is possible to estimate exact fragments size and their spatial distribution. Herein, fragmentation model is applied on the more idealistic situation where one cylindrical monolith rock specimen is blasted using one axially placed charge hole. In comparison with the blasting in the rock mass there are no pre-existing joints in the blasted medium. As it is well known pre-existing joints in the rock mass are limiting the propagation of the blast wave through the blasted medium. Therefore, radial tension cracks that are formed under the explosive load won't be limited in their length is blasted medium is monolith rock. Also, specimen has the "free surface" all around it, so it is assumed that tension cracks length will be equal in all directions. This makes it possible to compare model results with results of laboratory tests that were performed in same manner. One axial borehole in cylindrical rock specimen is filled with explosive and initiated. As a result of explosive detonation, radial tension cracks are formed. According to the rock 
blasting theory it is possible to calculate radii of zones with different density of radial cracks using Equation 15. Figure 3 and
Figure 4 illustrates rock specimen with formed radial tension cracks and different cracking zones.

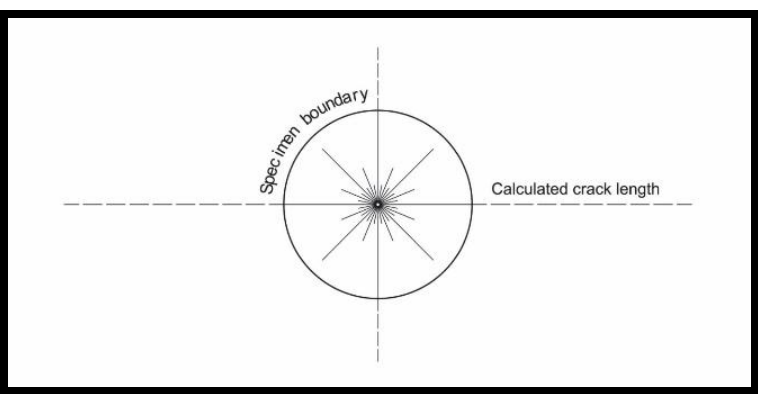

Figure 3 Relation between specimen and calculated crack dimensions

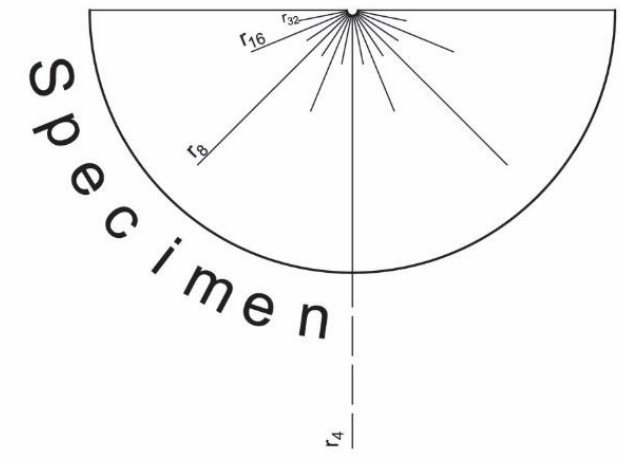

Figure 4 Detail of rock specimen with tension cracks

According to the previous assumptions, that fragments are quasi-isometric in their size with fractal like distribution, it is expected that fragmenting of the rock specimen is close to the idealistic fragmenting illustrated in Figure 5 and Figure 6.

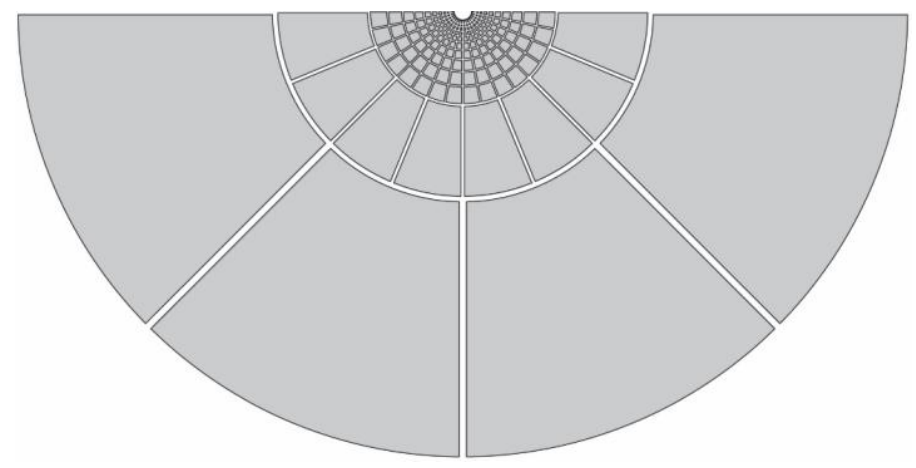

Figure 5 Fragmenting of rock specimen 

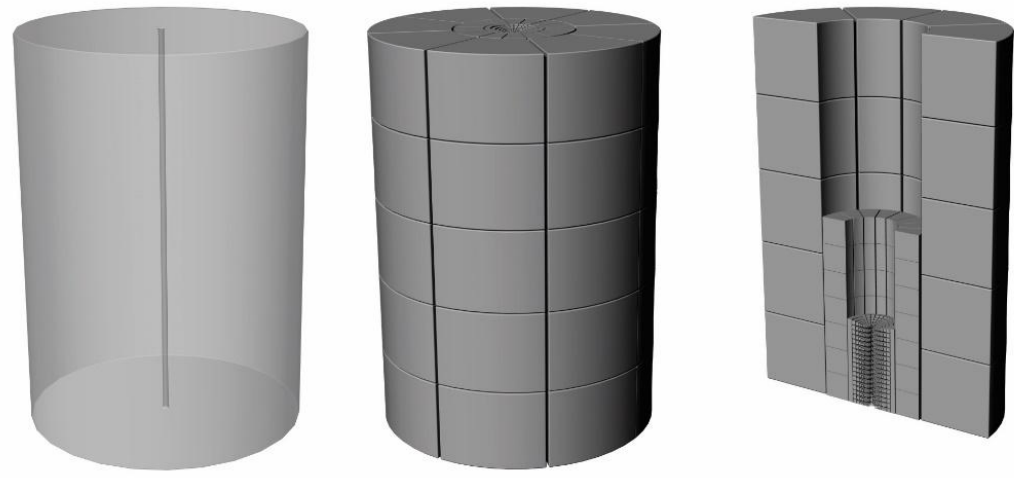

Figure $63 D$ illustration of specimen fragmenting

As it can be seen fragment dimensions are determined by crack density and radii of each cracking zone. Fragment length depends on the length difference between two adjacent cracking zones, while fragment width is determined directly by crack density in each cracking zone. Height of each fragment is equal to the maximum dimension between length and width. Fragment dimensions are illustrated in Figure 7.

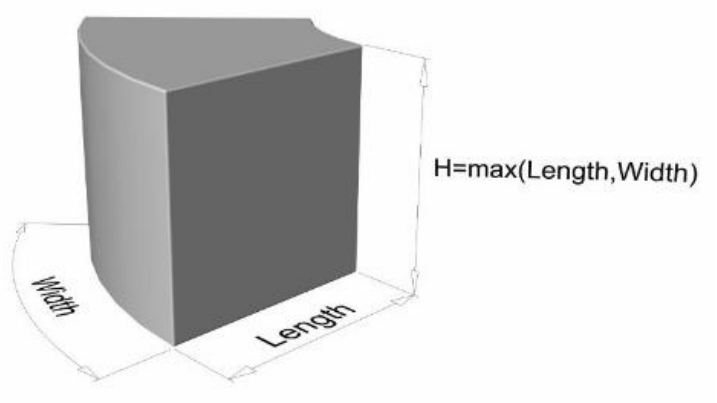

Figure 7 Fragment dimensions

\section{COMPARISON BETWEEN MODEL AND LABORATORY RESULTS}

Fragmentation model described in Section 3 was compared with lab-scale data [15]. Lab tests were conducted in the blast chamber in Leoben, Austria. Cylindrical rock specimens (Swedish Bårarp granite) were blasted using PETN explosive put into $5 \mathrm{~mm}$ diameter charge hole drilled axially through the sample; VOD was measured as control parameter. Figure 8 illustrates sample that is prepared for blasting. Tensile strength of rock is reported to be $13 \mathrm{MPa}$ while no data regarding Poisson's ratio was available and therefore $v=0.25$ is adopted for calculation. Table 2 presents sample data that was used in lab-scale tests. Results obtained from lab-scale tests are presented in Figure 9. 


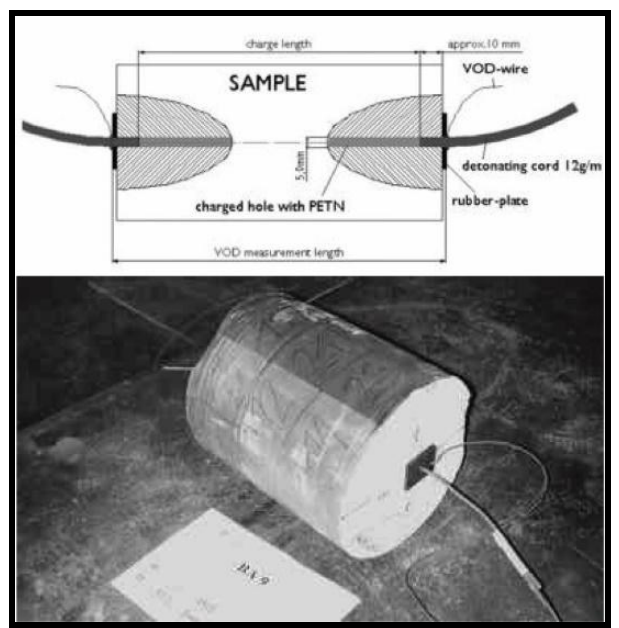

Figure 8 Sample prepared for blasting [15]

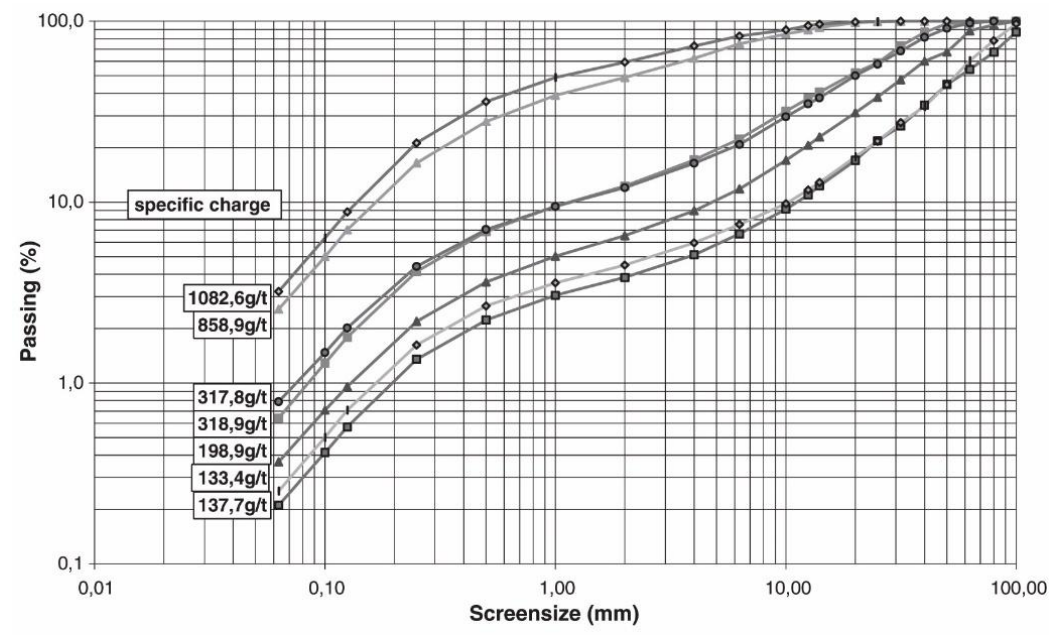

Figure 9 Particle size distribution from lab-scale tests [15]

Table 2 Data for lab-scale tests [15]

\begin{tabular}{lccccccc}
\hline Sample & $\begin{array}{c}\text { Diam. } \\
(\mathbf{m m})\end{array}$ & $\begin{array}{c}\text { Height } \\
(\mathbf{m m})\end{array}$ & $\begin{array}{c}\text { Mass } \\
(\mathbf{k g})\end{array}$ & $\begin{array}{c}\text { Charge } \\
(\mathbf{g})\end{array}$ & $\begin{array}{c}\text { Charge density } \\
\left(\mathrm{g} / \mathbf{c m}^{3}\right)\end{array}$ & $\begin{array}{c}\text { VOD } \\
(\mathbf{m} / \mathbf{s})\end{array}$ & $\begin{array}{c}\text { Spec. charge } \\
(\mathrm{g} / \text { ton })\end{array}$ \\
\hline BA9 & 289 & 333 & 58.10 & 7.75 & 1.11 & 5743 & 133.4 \\
BA2_2 & 192 & 310 & 24.04 & 7.64 & 1.18 & 5699 & 317.8 \\
BA1_1 & 243 & 355 & 43.38 & 8.63 & 1.17 & 5879 & 198.9 \\
BA10_2 & 103 & 218 & 4.84 & 5.24 & 1.19 & 5339 & 1082.6 \\
BA10_1 & 103 & 217 & 4.82 & 4.14 & 0.94 & 5135 & 858.9 \\
BA1_2 & 192 & 393 & 30.46 & 9.72 & 1.16 & 5842 & 319.0 \\
BA2_1 & 290 & 367 & 61.21 & 8.43 & 1.07 & 5459 & 137.7 \\
\hline
\end{tabular}


Calculation procedure for theoretical model is performed for each of the samples. Pressure inside the charge holes is calculated using measured VODs and reported charge densities. According to the ChapmanJouguet detonation theory $[16,17]$ pressure on the blast hole walls for explosives with density above $1 \mathrm{~g} / \mathrm{cm}^{3}$ can be calculated as:

$$
P_{d}=\frac{\rho_{\Omega}-D^{2}}{g}
$$

Where:

$$
\begin{aligned}
& P_{e}-\text { density of explosive }(\mathrm{g} / \mathrm{cm} 3) \\
& \mathrm{D} \text { - detonation velocity of explosive } \\
& (\mathrm{km} / \mathrm{s})
\end{aligned}
$$

For explosives with density below $1 \mathrm{~g} / \mathrm{cm}^{3}$ pressure on the blast hole walls is calculated by:

$$
P_{d}=\frac{\rho_{g} \cdot D^{2}}{4.5}
$$

Using calculated pressure and strength parameters of rock radii of each cracking zone is calculated at first. Then, fragments are drawn inside the sample boundaries and size of fragments in each of the zones is measured. Complete sample is divided into the fragments. Figure 10 a) presents reconstructed specimen after blasting [18] while b) presents fragmented rock specimen according to the presented model. Volume of the fragments is determined using CAD software and it is assumed that rock density is uniformly distributed in sample, so fragment volume and mass are proportional.

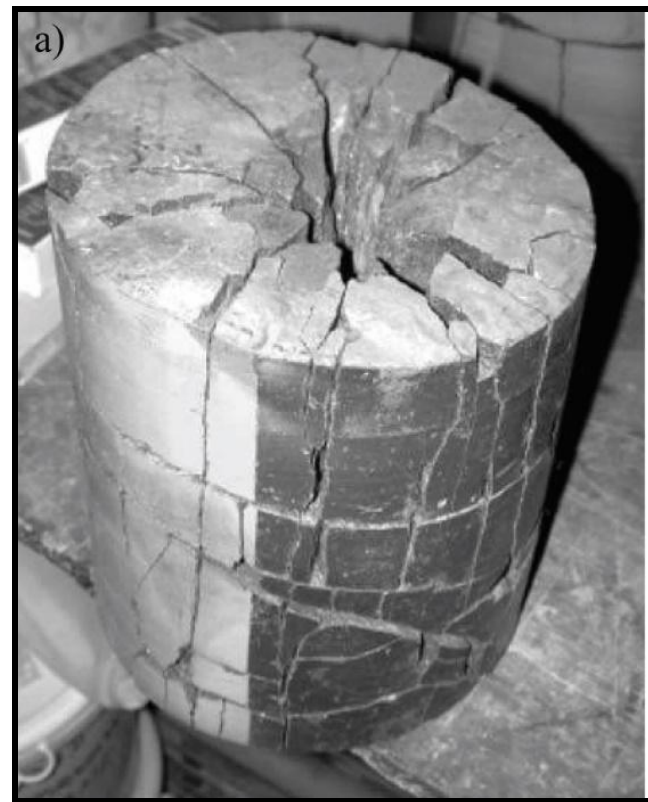

b)

Figure 10 a) reconstructed specimen after blasting [18] b) fragmented rock specimen according to the presented model

Fragment sizes are classified in the same size classes as results from lab-scale tests and correlation coefficient is calcu- lated for each sample. For each sample cumulative passing curves are plotted and shown in Figure 11 - Figure 18. 


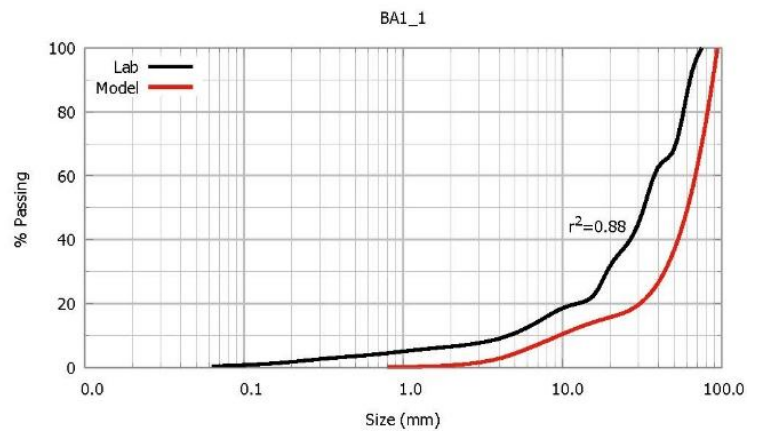

Figure 11 Results comparison for sample BA1_1

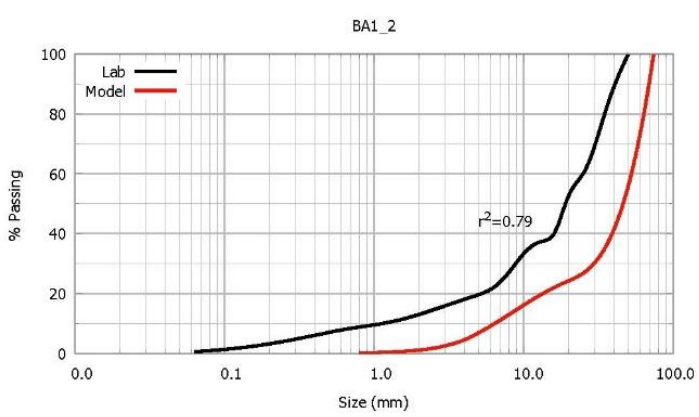

Figure 12 Results comparison for sample BA1_2

BA2 1

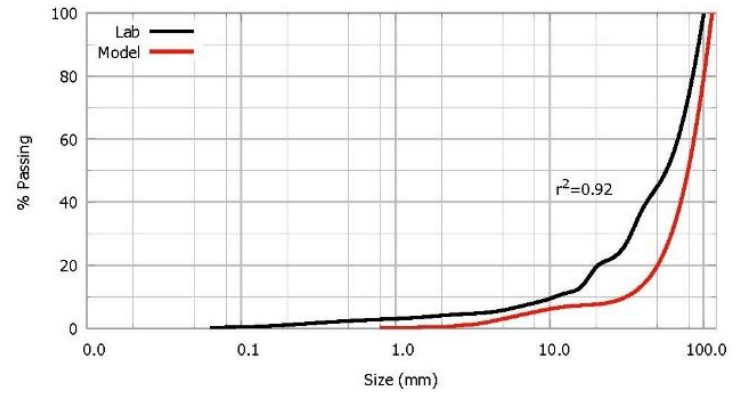

Figure 13 Results comparison for sample BA2_1

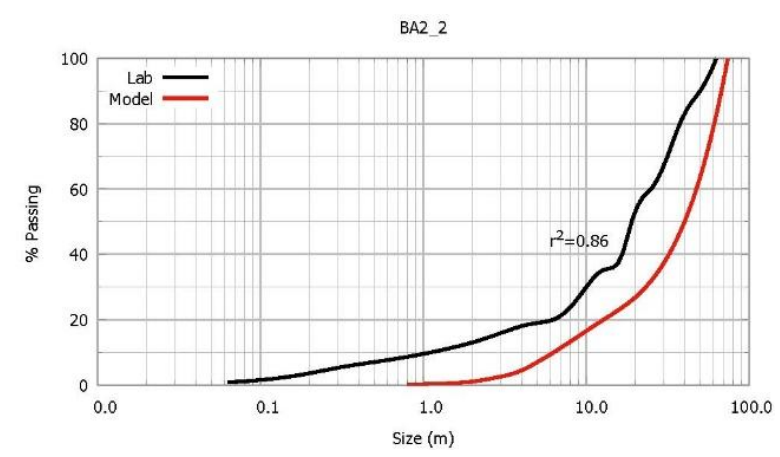

Figure 14 Results comparison for sample BA2_2 
BA9

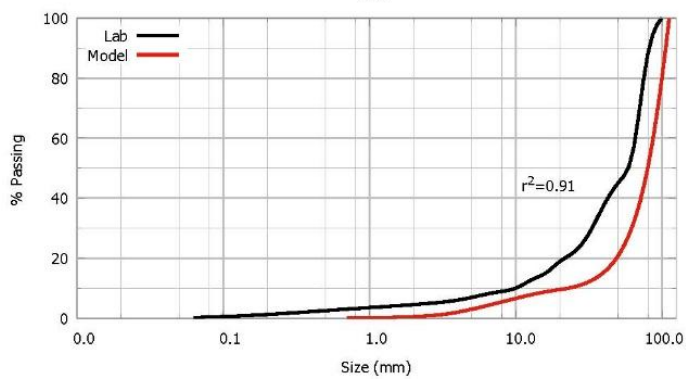

Figure 15 Results comparison for sample BA9

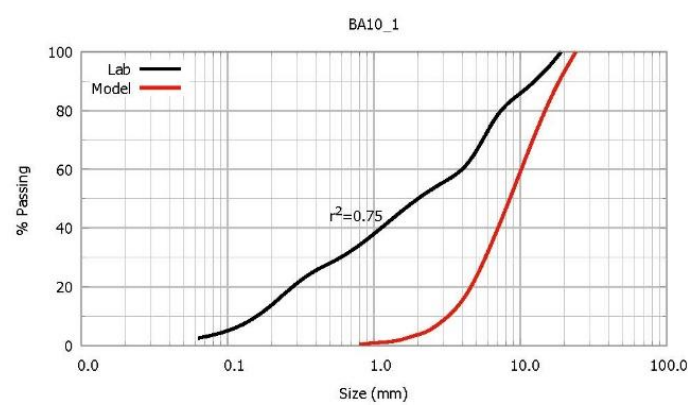

Figure 16 Results comparison for sample BA10_1

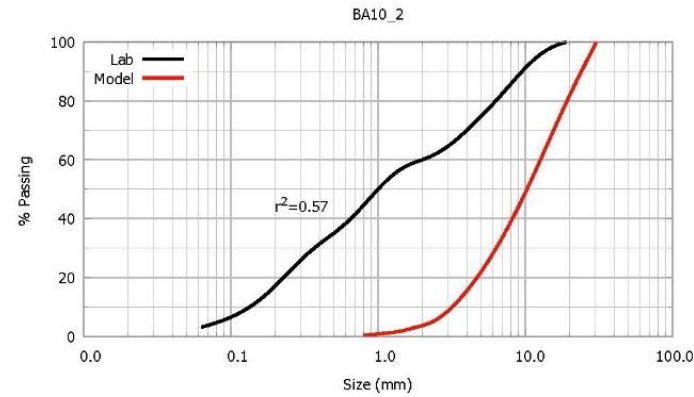

Figure 17 Results comparison for sample BA10_2

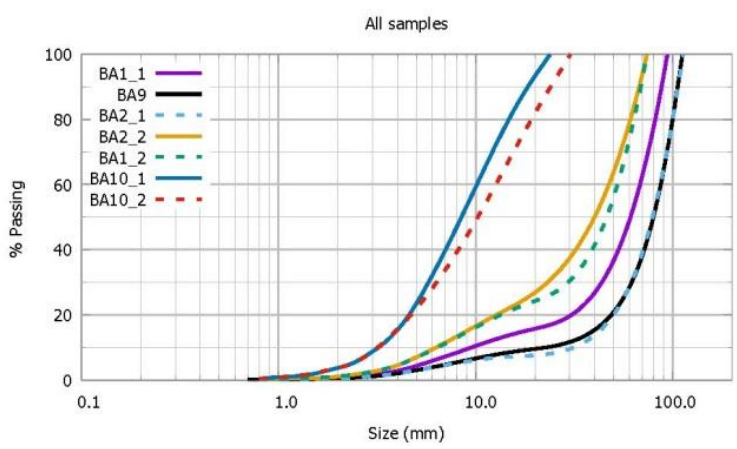

Figure 18 Model results comparison for all samples 
As it can be seen there is strong agreement between model and laboratory test results for samples BA1_1. BA1_2, BA2_1, BA2_2, BA_9 where correlation coefficient spans between 0.79 and 0.92 . In all of those cases model results show systematically larger fragment sizes in comparison to the laboratory ones. There are few possible reasons for this. As first, Poisson's ratio of rock is not known and value of 0.25 was adopted for calculation. Changing values from 0.2 and 0.3 , for same borehole pressure, gives different sizes of cracking zones radii. This means that fragment size distribution may be slightly different depending on the real Poisson's ratio value. On the other side, model was calculated using measured VOD values in order to maintain comparability of results and to eliminate any inconsistency related to the input data. Using the theoretical VOD values that can be calculated according to the charge densities would give slightly higher VOD values, which implies higher borehole pressure and the longer radii of the cracking zones. In this case it is to be expected that fragment sizes are smaller, which would make laboratory and model results much closer.

On the other side, results for the samples BA10_1 and BA10_2 show lower agreement between laboratory and model results. In those cases correlation coefficients are 0.75 and 0.57 , respectively. In both cases model results show higher fragment sizes. Reasons for this discrepancy of results may be different. In case of the sample BA10_1 charge density is below $1 \mathrm{~g} / \mathrm{cm}^{3}$ which imply application of the different equation for the pressure calculation (see eqn. 17). Therefore, there is possibility that discrepancy of the results in this case comes from the incorrect pressure estimate. Other possibility may encompass the strength heterogeneity between samples. Due to the small specimen dimensions slight change of the input data would result in appreciable changes of the fragment sizes. In the case of the specimen BA10_2 there is even greater discre- pancy of the results. However, it should be noted that in this case there is the highest charge density in comparison with all other samples while the VOD is significantly lower than it is expected. This means that borehole pressure may be incorrectly estimated which resulted in higher fragment sizes.

\section{REFERENCES}

[1] Kuznetsov, V. The Mean Diameter of the Fragments Formed by Blasting Rock. Journal of Mining Science, 9(2), (1973), 144-148.

[2] Cunningham, C. The Kuz-ram Model for the Prediction of Fragmentation from Blasting. In Proc. $1^{\text {st }}$ Intnl. Symp. Rock Fragmentation by Blasting, Lulea, (1983).

[3] Cunningham, C. Fragmentation Estimations and the Kuz-ram ModelFour Years on. In Proc. $2^{\text {nd }}$ Intnl. Symp. Rock Fragmentation by Blasting, Keystone, (1987).

[4] Ouchterlony, F., The Swebrecc Function: Linking Fragmentation by Blasting and Crushing. Mining Technology, 114(1), (2005), 29-44.

[5] Sanchidrián, J. A., Ouchterlony, F., Moser, P., Segarra, P., and López, L. M., Performance of Some Distributions to Describe Rock Fragmentation Data. International Journal of Rock Mechanics and Mining Sciences, 53, (2012), 18-31.

[6] Sanchidrián, J. A., Ouchterlony, F., Segarra, P., and Moser, P. (2014). Size Distribution Functions for Rock Fragments. International Journal of Rock Mechanics and Mining Sciences, 71:381-394.

[7] Djordjevic, N., A Two-component Model of Blast Fragmentation. In AusIMM Proceedings (Australia), Volume 304, (1999), pages 9-13.

[8] Ouchterlony, F., Prediction of Crack Lengths in Rock After Cautious 
Blasting With Zero Inter-hole Delay. Fragblast, 1(4), (1997), 417-444.

[9] Wang, Z., Konietzky, H., and Shen, R., Coupled Finite Element and Discrete Element Method for Underground Blast in Faulted Rock Masses. Soil Dynamics and Earthquake Engineering, 29(6), (2009), 939-945.

[10] Aliabadian, Z., Sharafisafa, M., Mortazavi, A., and Maarefvand, P. (2014). Wave and Fracture Propagation in Continuum and Faulted Rock Masses: Distinct Element Modeling. Arabian Journal of Geosciences, 7(12), (2009), 5021-5035.

[11] Torbica, S. and Lapcevic, V., Rock Breakage by Explosives. European International Journal of Science and Technology, (2014), p. 96-104.

[12] Mavko, G., Mukerji, T., and Dvorkin, J., The Rock Physics Handbook: Tools for Seismic Analysis of Porous Media. Cambridge University Press, 2009.

[13] Torbica, S., Lapcevic, V. Model for Estimating Blasted Rock Fragmentation. In: SGEM Geoconference on Science and Technologies. In: Geology, Explo-ration and Mining. www.sgem.org, SGEM2014 GeoConference Proceedings, ISBN 978-6197105-09-4 / ISSN 1314-2704, June 1925, 2014, v. 3, (2014), p. 379-386.
[14] Torbica, S. and Lapčević, V., Estimating Extent and Properties of Blastdamaged Zone Around Underground Excavations. Rem: Revista Escola de Minas, 68(4), (2015), 441-453.

[15] Moser, P., Olsson, M., Ouchterlony, F., and Grasedieck, A., Comparison of the Blast Fragmentation from Labscale and Full-scale Tests at Bårarp. In Proceedings of the EFEE $2^{\text {nd }}$ World Conference, Prague, Czech Republic, 10-12 September 2003, pages 449458. Informa UK Limited.

[16] Chapman, D. L., VI. on the Rate of Explosion in Gases. Philosophical Magazine Series 5, 47(284), (1899), 90-104.

[17] Jouguet, Sur la Propagation des Réactions Chimiques Dans les gaz. Journal de Mathématiques Pures et Appliquées, 1, (1905), 347-425.

[18] Moser, P., Grasedieck, A., du Mouza, J., and Hamdi, E., Breakage Energy in Rock Blasting. In Proceedings of the EFEE $2^{\text {nd }}$ World Conference, Prague, Czech Republic, 10-12 September 2003, p. 323-334. Informa UK Limited. 